\title{
The Formal Construction of Fuzzy Numbers
}

\author{
Adam Grabowski \\ Institute of Informatics \\ University of Białystok \\ Akademicka 2, 15-267 Białystok \\ Poland
}

\begin{abstract}
Summary. In this article, we continue the development of the theory of fuzzy sets 23], started with [14] with the future aim to provide the formalization of fuzzy numbers 8 in terms reflecting the current state of the Mizar Mathematical Library. Note that in order to have more usable approach in [14, we revised that article as well; some of the ideas were described in 12. As we can actually understand fuzzy sets just as their membership functions (via the equality of membership function and their set-theoretic counterpart), all the calculations are much simpler. To test our newly proposed approach, we give the notions of (normal) triangular and trapezoidal fuzzy sets as the examples of concrete fuzzy objects. Also $\alpha$-cuts, the core of a fuzzy set, and normalized fuzzy sets were defined. Main technical obstacle was to prove continuity of the glued maps, and in fact we did this not through its topological counterpart, but extensively reusing properties of the real line (with loss of generality of the approach, though), because we aim at formalizing fuzzy numbers in our future submissions, as well as merging with rough set approach as introduced in [13] and [1]. Our base for formalization was 9 and 10 .
\end{abstract}

MSC: 03E72 94D99 03B35

Keywords: fuzzy sets; formal models of fuzzy sets; triangular fuzzy numbers

MML identifier: FUZNUM_1, version: 8.1.03 5.29.1227

The notation and terminology used in this paper have been introduced in the following articles: [16], [3], 44, 5], [14, 2], 19], [1], 6], [17, 21, 22], 20], and [7. 


\section{Preliminaries: Affine Maps}

Now we state the proposition:

(1) Let us consider real numbers $a, b$. Suppose $a \leqslant b$. Then $\mathbb{R} \backslash] a, b[\neq \emptyset$.

From now on $a, b, c, x$ denote real numbers.

Now we state the propositions:

(2) $\quad\left(\operatorname{AffineMap}\left(\frac{1}{b-a},-\frac{a}{b-a}\right)\right)(a)=0$.

(3) If $b-a \neq 0$, then $\left(\operatorname{AffineMap}\left(\frac{1}{b-a},-\frac{a}{b-a}\right)\right)(b)=1$.

(4) If $c-b \neq 0$, then $\left(\operatorname{AffineMap}\left(-\frac{1}{c-b}, \frac{c}{c-b}\right)\right)(b)=1$.

(5) (AffineMap $\left.\left(-\frac{1}{c-b}, \frac{c}{c-b}\right)\right)(c)=0$.

(6) If $b-a \neq 0$ and $\left(\operatorname{AffineMap}\left(\frac{1}{b-a},-\frac{a}{b-a}\right)\right)(x)=1$, then $x=b$. The theorem is a consequence of $(3)$.

(7) If $c-b \neq 0$ and $\left(\operatorname{AffineMap}\left(-\frac{1}{c-b}, \frac{c}{c-b}\right)\right)(x)=1$, then $x=b$. The theorem is a consequence of (4).

(8) $\operatorname{rng}(\operatorname{AffineMap}(0, a))=\{a\}$.

(9) Let us consider a non empty subset $C$ of $\mathbb{R}$.

Then $\operatorname{rng}((\operatorname{AffineMap}(0, a))\lceil C)=\{a\}$.

Proof: Set $f=(\operatorname{AffineMap}(0, a))\lceil C \cdot \operatorname{rng} f \subseteq\{a\}$ by [3, (49)].

(10) If $b-a>0$, then $\operatorname{rng}\left(\left(\operatorname{AffineMap}\left(\frac{1}{b-a},-\frac{a}{b-a}\right)\right) \uparrow[a, b]\right)=[0,1]$.

Proof: Set $f=\operatorname{AffineMap}\left(\frac{1}{b-a},-\frac{a}{b-a}\right)$. Set $g=f\lceil[a, b]$. rng $g \subseteq[0,1]$ by [21, (57)], [3, (47)], (2), [16, (53)].

Let us assume that $c-b>0$. Now we state the propositions:

(11) $\left.\operatorname{rng}\left(\left(\operatorname{AffineMap}\left(-\frac{1}{c-b}, \frac{c}{c-b}\right)\right)\lceil] b, c\right]\right)=[0,1[$.

Proof: Set $f=\operatorname{AffineMap}\left(-\frac{1}{c-b}, \frac{c}{c-b}\right)$. Set $\left.g=f\lceil] b, c\right] . \operatorname{rng} g \subseteq[0,1[$ by [21, (57)], [3, (47)], (4), [16, (52), (54)].

(12) $\operatorname{rng}\left(\left(\operatorname{AffineMap}\left(-\frac{1}{c-b}, \frac{c}{c-b}\right)\right) \uparrow[b, c]\right)=[0,1]$.

Proof: Set $f=\operatorname{AffineMap}\left(-\frac{1}{c-b}, \frac{c}{c-b}\right)$. Set $g=f\lceil[b, c] \cdot \operatorname{rng} g \subseteq[0,1]$ by [21, (57)], [3, (47)], (4), [16, (54)].

Now we state the propositions:

(13) $(\operatorname{AffineMap}(0,0))(x) \neq 1$.

(14) $(\operatorname{AffineMap}(0,1))(b)=1$.

(15) Let us consider a real number $a$. Then $(\operatorname{AffineMap}(0, b))(a)=b$. 


\section{Towards Development of Fuzzy Numbers}

In the sequel $C$ denotes a non empty set.

Let $C$ be a non empty set.

A fuzzy set of $C$ is a membership function of $C$. Let $F$ be a fuzzy set of $C$. We say that $F$ is normalized if and only if

(Def. 1) there exists an element $x$ of $C$ such that $F(x)=1$.

We introduce $F$ is normal as a synonym of $F$ is normalized.

We introduce $F$ is subnormal as an antonym for $F$ is normal.

We say that $F$ is strictly normalized if and only if

(Def. 2) there exists an element $x$ of $C$ such that $F(x)=1$ and for every element $y$ of $C$ such that $F(y)=1$ holds $y=x$.

One can verify that every fuzzy set of $C$ which is strictly normalized is also normalized.

Let $F$ be a fuzzy set of $C$ and $\alpha$ be a real number. The functor $\alpha$-cut $(F)$ yielding a subset of $C$ is defined by the term

(Def. 3) $\{x$, where $x$ is an element of $C: F(x) \geqslant \alpha\}$.

Now we state the proposition:

(16) Let us consider a fuzzy set $F$ of $C$, and a real number $\alpha$. Then $\alpha$-cut $(F)=$ $F^{-1}([\alpha, 1])$.

Proof: $\alpha-\operatorname{cut}(F) \subseteq F^{-1}([\alpha, 1])$ by $[6,(4)]$.

Let us consider $C$. Let us note that UMF $C$ is normalized and there exists a fuzzy set of $C$ which is normalized.

Let $F$ be a fuzzy set of $C$. The functor Core $F$ yielding a subset of $C$ is defined by the term

(Def. 4) $\{x$, where $x$ is an element of $C: F(x)=1\}$.

Now we state the propositions:

(17) Core UMF $C=C$.

(18) Core $\operatorname{EMF} C=\emptyset$.

Let us consider $C$. One can check that Core EMF $C$ is empty.

Let us consider a fuzzy set $F$ of $C$. Now we state the propositions:

(19) Core $F=F^{-1}(\{1\})$.

(20) Core $F=1-\operatorname{cut}(F)$. The theorem is a consequence of (16) and (19). 


\section{Convexity and the Height of a Fuzzy Set}

Let $F$ be a fuzzy set of $\mathbb{R}$. We say that $F$ is convex if and only if

(Def. 5) for every real numbers $x_{1}, x_{2}$ and for every real number $l$ such that $0 \leqslant l \leqslant 1$ holds $F\left(l \cdot x_{1}+(1-l) \cdot x_{2}\right) \geqslant \min \left(F\left(x_{1}\right), F\left(x_{2}\right)\right)$.

Observe that UMF $\mathbb{R}$ is convex and EMF $\mathbb{R}$ is convex.

Let $C$ be a non empty set and $F$ be a fuzzy set of $C$. The functor height $F$ yielding an extended real is defined by the term

(Def. 6) $\sup \operatorname{rng} F$.

Now we state the propositions:

(21) Let us consider a fuzzy set $F$ of $C$. Then $0 \leqslant$ height $F \leqslant 1$.

Proof: 0 is a lower bound of $\operatorname{rng} F$ by [15, (1)]. 1 is a upper bound of $\operatorname{rng} F$ by [15, (1)].

(22) Let us consider a fuzzy set $F$ of $C$. If $F$ is normalized, then height $F=1$. The theorem is a consequence of (21).

\section{Pasting aka Glueing Lemmas}

Let us consider partial functions $f, g$ from $\mathbb{R}$ to $\mathbb{R}$. Now we state the proposition:

(23) Suppose $f$ is continuous and $g$ is continuous and there exists an object $x$ such that $\operatorname{dom} f \cap \operatorname{dom} g=\{x\}$ and for every object $x$ such that $x \in$ $\operatorname{dom} f \cap \operatorname{dom} g$ holds $f(x)=g(x)$. Then there exists a partial function $h$ from $\mathbb{R}$ to $\mathbb{R}$ such that

(i) $h=f+\cdot g$, and

(ii) for every real number $x$ such that $x \in \operatorname{dom} f \cap \operatorname{dom} g$ holds $h$ is continuous in $x$.

Proof: Reconsider $h=f+\cdot g$ as a partial function from $\mathbb{R}$ to $\mathbb{R}$. For every real number $r$ such that $0<r$ there exists a real number $s$ such that $0<s$ and for every real number $x_{1}$ such that $x_{1} \in \operatorname{dom} h$ and $\left|x_{1}-x\right|<s$ holds $\left|h\left(x_{1}\right)-h(x)\right|<r$ by [21, (57)], [16, (3)], [5, (12)], [3, (47)].

Let us assume that $f$ is continuous and non empty and $g$ is continuous and non empty and there exist real numbers $a, b, c$ such that $\operatorname{dom} f=[a, b]$ and $\operatorname{dom} g=[b, c]$ and $f \approx g$. Now we state the propositions:

(24) There exists a partial function $h$ from $\mathbb{R}$ to $\mathbb{R}$ such that

(i) $h=f+\cdot g$, and 
(ii) for every real number $x$ such that $x \in \operatorname{dom} h$ holds $h$ is continuous in $x$.

(25) $f+\cdot g$ is continuous. The theorem is a consequence of (24).

Now we state the proposition:

(26) Suppose $g$ is not empty and $f=(\operatorname{AffineMap}(0,0)) \uparrow(\mathbb{R} \backslash] a, b[)$ and $\operatorname{dom} g=$ $[a, b]$ and $g(a)=0$ and $g(b)=0$. Then $f \approx g$.

PRoOF: For every object $x$ such that $x \in \operatorname{dom} f \cap \operatorname{dom} g$ holds $f(x)=g(x)$ by [18, (1)], [3, (47)], (15).

Let us assume that $g$ is continuous and non empty and

$f=(\operatorname{AffineMap}(0,0)) \uparrow(\mathbb{R} \backslash] a, b[)$ and $\operatorname{dom} g=[a, b]$ and $g(a)=0$ and $g(b)=0$. Now we state the propositions:

(27) There exists a partial function $h$ from $\mathbb{R}$ to $\mathbb{R}$ such that

(i) $h=f+\cdot g$, and

(ii) for every real number $x$ such that $x \in \operatorname{dom} h$ holds $h$ is continuous in $x$.

The theorem is a consequence of (26).

(28) $f+\cdot g$ is continuous. The theorem is a consequence of (27).

Note that there exists a subset of $\mathbb{R}$ which is non trivial, closed interval, and closed.

\section{Triangular and Trapezoidal Fuzzy Sets}

Let $a, b, c$ be real numbers. Assume $a<b$ and $b<c$.

The functor TriangularFS $(a, b, c)$ yielding a fuzzy set of $\mathbb{R}$ is defined by the term

(Def. 7) ((AffineMap $(0,0)) \uparrow(\mathbb{R} \backslash] a, c[)+$.

$\left.\left(\operatorname{AffineMap}\left(\frac{1}{b-a},-\frac{a}{b-a}\right)\right) \uparrow[a, b]\right)+$.

(AffineMap $\left.\left(-\frac{1}{c-b}, \frac{c}{c-b}\right)\right) \uparrow[b, c]$.

Let us consider real numbers $a, b, c$. Let us assume that $a<b<c$. Now we state the propositions:

(29) TriangularFS $(a, b, c)$ is strictly normalized.

Proof: Set $F=$ TriangularFS $(a, b, c)$. Reconsider $b_{1}=b$ as an element of $\mathbb{R}$. For every element $y$ of $\mathbb{R}$ such that $F(y)=1$ holds $y=b_{1}$ by [21, (57)], [5, (11), (13)], [3, (49)].

(30) TriangularFS $(a, b, c)$ is continuous.

Proof: Set $f_{1}=\operatorname{AffineMap}(0,0)$. Set $f=f_{1} \uparrow(\mathbb{R} \backslash] a, c[)$. Set $g_{1}=$ AffineMap $\left(\frac{1}{b-a},-\frac{a}{b-a}\right)$. Reconsider $g=g_{1} \uparrow[a, b]$ as a partial function from 
$\mathbb{R}$ to $\mathbb{R}$. Set $h_{1}=\operatorname{AffineMap}\left(-\frac{1}{c-b}, \frac{c}{c-b}\right)$. Reconsider $h=h_{1} \uparrow[b, c]$ as a partial function from $\mathbb{R}$ to $\mathbb{R}$. For every object $x$ such that $x \in \operatorname{dom} g \cap \operatorname{dom} h$ holds $g(x)=h(x)$ by [3, (49)], (4), (3). Set $\mathfrak{h}=g+\cdot h$. Consider $h_{2}$ being a partial function from $\mathbb{R}$ to $\mathbb{R}$ such that $h_{2}=f+\mathfrak{h}$ and for every real number $x$ such that $x \in \operatorname{dom} h_{2}$ holds $h_{2}$ is continuous in $x$.

Let $a, b, c, d$ be real numbers. Assume $a<b$ and $b<c$ and $c<d$. The functor TrapezoidalFS $(a, b, c, d)$ yielding a fuzzy set of $\mathbb{R}$ is defined by the term

(Def. 8) $\quad((($ AffineMap $(0,0))\lceil(\mathbb{R} \backslash] a, d[)+$.

(AffineMap $\left.\left(\frac{1}{b-a},-\frac{a}{b-a}\right)\right)\lceil[a, b])+$.

$(\operatorname{AffineMap}(0,1)) \uparrow[b, c])+\cdot\left(\operatorname{AffineMap}\left(-\frac{1}{d-c}, \frac{d}{d-c}\right)\right) \uparrow[c, d]$.

Let us consider real numbers $a, b, c, d$. Let us assume that $a<b<c<d$. Now we state the propositions:

(31) TrapezoidalFS $(a, b, c, d)$ is normalized. The theorem is a consequence of (4).

(32) TrapezoidalFS $(a, b, c, d)$ is continuous.

Proof: Set $f_{1}=\operatorname{AffineMap}(0,0)$. Set $f=f_{1} \uparrow(\mathbb{R} \backslash] a, d[)$. Set $g_{1}=$ AffineMap $\left(\frac{1}{b-a},-\frac{a}{b-a}\right)$. Reconsider $g=g_{1}\lceil[a, b]$ as a partial function from $\mathbb{R}$ to $\mathbb{R}$. Set $h_{1}=\operatorname{AffineMap}\left(-\frac{1}{d-c}, \frac{d}{d-c}\right)$. Reconsider $h=h_{1}\lceil[c, d]$ as a partial function from $\mathbb{R}$ to $\mathbb{R}$. Set $i_{1}=\operatorname{AffineMap}(0,1)$. Reconsider $i=i_{1} \uparrow[b, c]$ as a partial function from $\mathbb{R}$ to $\mathbb{R}$. For every object $x$ such that $x \in \operatorname{dom} g \cap \operatorname{dom} i$ holds $g(x)=i(x)$ by [3, (49)], (15), (3). Set $\mathfrak{h}=g+\cdot i . \mathfrak{h}$ is continuous. For every object $x$ such that $x \in \operatorname{dom} \mathfrak{h} \cap \operatorname{dom} h$ holds $\mathfrak{h}(x)=h(x)$ by [5, (13)], 3, (49)], (15). Set $g_{2}=\mathfrak{h}+\cdot h$. Consider $h_{2}$ being a partial function from $\mathbb{R}$ to $\mathbb{R}$ such that $h_{2}=f+\cdot g_{2}$ and for every real number $x$ such that $x \in \operatorname{dom} h_{2}$ holds $h_{2}$ is continuous in $x$.

Let $F$ be a fuzzy set of $\mathbb{R}$. We say that $F$ is triangular if and only if

(Def. 9) there exist real numbers $a, b, c$ such that $F=\operatorname{TriangularFS}(a, b, c)$.

We say that $F$ is trapezoidal if and only if

(Def. 10) there exist real numbers $a, b, c, d$ such that $F=\operatorname{TrapezoidalFS}(a, b, c, d)$.

One can verify that there exists a fuzzy set of $\mathbb{R}$ which is triangular and there exists a fuzzy set of $\mathbb{R}$ which is trapezoidal.

\section{REFERENCES}

[1] Grzegorz Bancerek. The ordinal numbers Formalized Mathematics, 1(1):91-96, 1990.

[2] Józef Białas. Properties of the intervals of real numbers Formalized Mathematics, 3(2): 263-269, 1992.

[3] Czesław Byliński. Functions and their basic properties Formalized Mathematics, 1(1): 55-65, 1990. 
[4] Czesław Byliński. Functions from a set to a set Formalized Mathematics, 1(1):153-164, 1990.

[5] Czesław Byliński. The modification of a function by a function and the iteration of the composition of a function. Formalızed Mathematıcs, 1(3):521-527, 1990.

[6] Czesław Byliński. Partial functions. Formalized Mathematics, 1(2):357-367, 1990.

[7] Czesław Byliński. Some basic properties of sets Formalized Mathematics, 1(1):47-53, 1990.

[8] Didier Dubois and Henri Prade. Operations on fuzzy numbers. International Journal of System Sciences, 9(6):613-626, 1978.

[9] Didier Dubois and Henri Prade. Fuzzy Sets and Systems: Theory and Applications. Academic Press, New York, 1980.

[10] Didier Dubois and Henri Prade. Rough fuzzy sets and fuzzy rough sets. International Journal of General Systems, 17(2-3):191-209, 1990.

[11] Adam Grabowski. Efficient rough set theory merging. Fundamenta Informaticae, 135(4): 371-385, 2014. doi $10.3233 /$ FI-2014-1129.

[12] Adam Grabowski. On the computer certification of fuzzy numbers. In M. Ganzha, L. Maciaszek, and M. Paprzycki, editors, 2013 Federated Conference on Computer Science and Information Systems (FedCSIS), Federated Conference on Computer Science and Information Systems, pages 51-54, 2013.

[13] Adam Grabowski. Basic properties of rough sets and rough membership function. Formalized Mathematics, 12(1):21-28, 2004.

[14] Takashi Mitsuishi, Noboru Endou, and Yasunari Shidama. The concept of fuzzy set and membership function and basic properties of fuzzy set operation Formallzed Mathematıcs, 9(2):351-356, 2001.

[15] Takashi Mitsuishi, Katsumi Wasaki, and Yasunari Shidama. Basic properties of fuzzy set operation and membership function. Formalized Mathematics, y(2):357-362, 2001.

[16] Konrad Raczkowski and Paweł Sadowski. Real function continuity. Formalized Mathematics, 1(4):787-791, 1990.

[17] Konrad Raczkowski and Paweł Sadowski. Topological properties of subsets in real numbers Formalized Mathematics, 1(4):777-780, 1990.

[18] Andrzej Trybulec. Enumerated sets. Formalized Mathematics, 1(1):25-34, 1990.

[19] Andrzej Trybulec. On the sets inhabited by numbers. Formalized Mathematics, 11(4): 341-347, 2003.

[20] Zinaida Trybulec. Properties of subsets Formalized Mathematics, 1(1):67-71, 1990.

[21] Edmund Woronowicz. Relations and their basic properties Formalized Mathematics, 1 (1):73-83, 1990.

[22] Edmund Woronowicz. Relations defined on sets. Formalized Mathematics, 1(1):181-186, 1990.

[23] Lotfi Zadeh. Fuzzy sets. Information and Control, 8(3):338-353, 1965.

Received December 31, 2014 